programmes where trainees from the UK come to Nigeria to experience psychiatric practice. It would facilitate the exchange of ideas and they may pick up ingenious ways to solve complex problems in the absence of robust mental health legislation. This would need consideration by local UK trusts and deaneries and Health Education England to assure appropriateness of placement, training specifications and overall goal of the exercise. It might also be useful for senior UK psychiatrists, especially those who supervise MTI trainees, to spend some time in Nigeria. They would learn about the complexities of practice and might even begin to understand what the country's training encompasses to enable progress in placements, so that Nigerian MTI trainees are not allocated to posts that are way below their level of expertise.

\section{Data availability}

Data availability is not applicable to this article as no new data were created or analysed in this study.

\section{Author contributions}

N.I. and B.M. contributed Nigerian perspectives on Forensic Psychiatry training and practice and MTI trainee experience. J.L. and D.T. contributed perspectives on UK Forensic psychiatry training and practice.

\section{Funding}

This research received no specific grant from any funding agency, commercial or not-for-profit sectors.

\section{Declaration of interest None.}

\section{References}

1 Royal College of Psychiatrists. Guidance for Trusts Employing an RCPsych MTI International Fellow. RCPsych, 2019 (https://www. rcpsych.ac.uk/docs/default-source/training/medical-training-initiative/ rcpsych-mti-trust-guidance-2019.pdf).

2 Arboleda-Florez J. Forensic psychiatry: contemporary scope, challenges and controversies. World Psychiatry 2006; 5: 87-91.

3 Ogunlesi AO, Ogunwale A. Correctional psychiatry in Nigeria: dynamics of mental healthcare in the most restrictive alternative. BJPsych Int 2018; 15: 35-8.

4 Federal Ministry of Justice. Criminal Procedure Act [Nigeria], Cap C41 Vol. 4. Federal Ministry of Justice, 2004.

5 Royal College of Psychiatrists. Routes to registration. RCPsych, 2021 (https://www.rcpsych.ac.uk/training/your-training/routes-toregistration)

6 Royal College of Psychiatrists. A Competency Based Curriculum for Specialist Training in Psychiatry: Specialists in General Psychiatry. RCPsych, 2019 (https://www.rcpsych.ac.uk/docs/default-source/ training/curricula-and-guidance/general_psychiatry_curriculum_march_ 2019.pdf)

7 Ogunlesi AO, Ogunwale A, De Wet P, Roos L, Kaliski S. Forensic psychiatry in Africa: prospects and challenges. Afr J Psychiatry 2012; 15: 3-7.

8 Abdalla-Filho E, Bertolote JM. Forensic psychiatric systems in the world. Rev Bras Psiquiatr 2006; 28: 56-61.

9 Igbinomwanhia NG, Akanni O. Constraints, ethical dilemmas and precautions in psychiatric practice within non-contemporaneous mental health laws: a Nigerian experience with involuntary commitment. J Law Policy Glob 2019; 87: 21-5.

10 Westbrook AH. Mental health legislation and involuntary commitment in Nigeria: a call for reform. Wash U Global Law Rev 2011; 10: 397-418.

11 Lagunes-Cordoba E, Maitra R, Dave S, Matheiken S, Oyebode F, O'Hara J, et al International medical graduates: how can UK psychiatry do better? BJPsych Bull 2021; 45: 299-304.

12 Academy of Medical Royal Colleges. Medical Training Initiative: Relocation Guide. Academy of Medical Royal Colleges, 2018 (https://www.aomrc.org.uk/wp-content/uploads/2018/05/MTI Relocation_Guide_MAY2018-v3-wr-1.pdf).

\title{
THEMATIC PAPER \\ Perinatal mental health around the world: priorities for research and service development in The Netherlands
}

\author{
Iris Leppers, ${ }^{1} \odot$ Cornelis Pieter Matthijs Veth, ${ }^{2}$ Dieuwertje Anna de Waardt, ${ }^{2}$ \\ Hanneke Migchels ${ }^{3}$ and Maria Johanna Traa ${ }^{4}$
}

${ }^{1} \mathrm{MD}$, Psychiatry Resident, Department of Psychiatry, Elisabeth-Tweesteden Hospital, Tilburg, The Netherlands. Email ileppers@etzn

${ }^{2} \mathrm{MD}$, Specialist Psychiatrist, Department of Psychiatry,

Elisabeth-Tweesteden Hospita, Tilburg, The Netherlands

${ }^{3} \mathrm{MD}$, Specialist Obstetrician, Department of Obstetrics and Gynaecology, ElisabethTweesteden Hospital, Tilburg, The
The Netherlands has an unique system of perinatal and postpartum healthcare. Pregnancy care is delivered predominantly by primary care midwives and childbirth services predominantly involve hospital care. The first week postpartum, all women receive daily care from maternity nurses. In addition, hospitals and out-patient clinics offer perinatal mental healthcare. More specifically, 'POP care' (psychiatry, obstetrics and paediatrics) was developed to promote multidisciplinary collaboration in this regard. Although clinical practices and government initiatives to improve pregnancy-related mental healthcare work well, they have yet to be fully described and evaluated. The current COVID-19 pandemic has an impact on health services and perinatal mental health. 
${ }^{4} \mathrm{PhD}$, Healthcare Psychologist, Department of Medical Psychology, ElisabethTweesteden Hospital, Tilburg, The Netherlands

Keywords. Perinatal psychiatry; depressive disorders; community mental health teams; bipolar affective disorders; epidemiology.

First received 14 Jul 2021 Final revision 5 Aug 2021 Accepted 9 Aug 2021

doi:10.1192/bji.2021.47

(c) The Author(s), 2021. Published by Cambridge University Press on behalf of the Royal College of Psychiatrists. This is an Open Access article, distributed under the terms of the Creative Commons Attribution licence (https://creativecommons.org/ licenses/by/4.0/), which permits unrestricted re-use, distribution, and reproduction in any medium rovided the original work properly cited.
The Netherlands, a densely populated country in Western Europe, has about 17 million inhabitants (416 persons per square kilometre) and had 168 525 live births (relatively 9.8 per 1000 inhabitants) in 2018. The Netherlands is often perceived as a modern and tolerant society, referring to the multicultural composition of its population. In $2020,24.4 \%$ of the total population had a migration background, including first- and secondgeneration immigrants. Most of them originate from Turkey and Morocco. Within Europe, The Netherlands has been rated the highest 3 years in a row for satisfaction of inhabitants with their healthcare system and the accessibility of healthcare and medications. Within The Netherlands, mental healthcare, however, does differ from one region to the next. ${ }^{1}$

There has been an increased interest in perinatal mental health during recent decades in The Netherlands. For instance, in 2009 the National Knowledge Centre for Psychiatry and Pregnancy was established to improve mental healthcare in the perinatal period.

The current paper starts by outlining current Dutch clinical practice of perinatal healthcare, followed by a description of the research on perinatal mental healthcare with recommendations for future improvements. Finally, the impact of the coronavirus disease 2019 (COVID-19) pandemic on perinatal mental health services is outlined.

\section{Dutch clinical practice \\ General organisation of healthcare services}

The Dutch government is responsible for the quality and accessibility of the country's healthcare system. The health policy and its implementation are organised at a national level by the Ministry of Health, Welfare and Sport. Under the ministry's responsibility, there is a basic health insurance, which is mandatory for all citizens older than 18 years. Government assistance is available for lowincome individuals and those unable to afford the basic health-insurance premium, thereby ensuring access to healthcare for all citizens. In our healthcare system the general practitioner (GP) functions as the main gatekeeper to specialised healthcare (e. g. hospital care), with the goal of enhancing the quality of healthcare and preventing excessive costs. Perinatal healthcare is available for all women, at no extra cost.

A decade ago, research suggested that healthcare use varied across different ethnic groups (with first-generation immigrants forming the largest minority). ${ }^{2}$ More recent research indicates that ethnic minorities visit the GP more often than the indigenous population. ${ }^{3}$ The current expansion of second-generation immigrants might lead to increased equality in healthcare use, as they are more integrated in society.

\section{Perinatal and postpartum care}

Integrated obstetric healthcare is provided. Pregnancy care is delivered predominantly by primary care midwives, who monitor pregnant women for physical and mental health problems, and make specific referrals if needed. So midwives function as gatekeepers during pregnancy. In 2016, the Standard for Integrated Birth Care was developed, which outlines the content and organisation of birth care in The Netherlands; if it is not medically necessary to give birth in a hospital, alternative locations are available: at home, in a birthing centre or in an out-patient clinic. In 2018, approximately $28 \%$ of all labours were supervised by primary care midwives and 13\% of all labours took place at home. The amount of childbirth at home is considerably higher in The Netherlands than in other European countries, where less than $1 \%$ of all labours took place at home. Perinatal mortality in The Netherlands is 4.2 per 1000 births (2015), and compared with other European countries The Netherlands holds the middle position. Dutch research showed no difference in the rates of intrapartum and neonatal death up to 28 days after birth between planned hospital births and planned home births among low-risk women. ${ }^{4}$ Analysis of Euro-PERISTAT data shows that the perinatal mortality rate at term is not significantly different in The Netherlands than in other European countries (in which home births and primary care births are uncommon). ${ }^{5}$ As the 'Dutch home birth culture' is unique in the world, this culture was added to the list of Dutch intangible heritage in 2020.

As mentioned above, all mothers are entitled to daily healthcare during the first week after delivery. The maternity nurse performs healthcare checks for both mother and neonate, informs and helps parents with common problems during the postpartum period (including mental health problems), is alert to problems in mother-baby interaction and supports the household. After maternity care, preventive healthcare in child health clinics follows, where the child's development is monitored as well as the mother-child relationship and maternal mental health. New mothers can download a mobile app, produced by the Municipal Health Service (GGD), to follow the child's development (growth curves, vaccinations, milestones, etc.).

Throughout pregnancy and the postpartum period, professionals are mandated to report suspicions of domestic violence and/or child abuse, in accordance with the Protocol of Domestic Violence and Child Abuse. Prevalence of intimate partner violence in the perinatal period ranges from 3.9 to $8.3 \% .^{6}$ Domestic violence is known to increase the risk of postpartum depression and suicidal ideation in new mothers, which can lead to problems in mother-child interaction and other threats. ${ }^{7}$ Signalling abuse is of great importance to optimise a healthy and safe environment for both mother and child.

The protocol additionally includes advice on informing the Safe at Home Centre. If the Safe at Home Centre is notified, this facility investigates and, if needed, initiates additional care. 


\section{Perinatal and postpartum mental healthcare}

As mentioned, a substantial part of perinatal care is delivered by midwives, who are trained to be aware of perinatal mental health. Throughout the country, various hospitals and out-patient clinics offer perinatal mental healthcare. In the past decade, considerable advancement has been achieved in 'POP care' (psychiatry, obstetrics and paediatrics). The POP team often includes psychologists, psychiatrists, gynaecologists, midwifes, paediatricians and social workers, which all strive to achieve excellent biopsychosocial healthcare through multidisciplinary collaboration. Referrals to POP teams come from the various collaborating disciplines, as well as from primary healthcare (GPs) and mental healthcare institutions. Psychiatry residents can choose to follow an internship focusing on perinatal mental healthcare during their training. Within POP care various treatment options can be offered, including (but not limited to) pre-conception counselling, psychological treatment (e.g. cognitivebehavioural therapy, eye-movement desensitisation and reprocessing) and/or pharmacological treatment. Postpartum care is also extensive. For instance, mothers with severe psychiatric illness and their infants can be admitted to specialised medical-psychiatric departments in hospitals, e.g. in-patient mother and baby units. These facilities provide psychiatric care for the mother and evaluate mother-baby interaction. More recently, infant mental health specialists - who are trained to evaluate and enhance mother-child interactions and infant mental health development - are increasingly participating in POP care. To support best practice for healthcare workers, evidence-based national guidelines are available concerning the use of psychiatric drugs (such as benzodiazepines and antidepressants) during the pre-conception period, pregnancy, childbirth and in the postpartum period. ${ }^{8}$

\section{Research on perinatal and postpartum mental healthcare in The Netherlands Perinatal}

Overall lifetime prevalence figures for mood and anxiety disorders in The Netherlands are 20.2\% and $19.6 \%$ respectively. ${ }^{9}$ The prevalence of depressive symptoms during pregnancy increased from $7 \%$ in 1988 to $14 \%$ in $2014 .{ }^{10}$ This is comparable to meta-analysed international rates by trimester: $7.4 \%$ (first trimester), $12.8 \%$ (second) and $12.0 \%$ (third). ${ }^{11}$ Two in five pregnant women with a history of major depression had at least one depressive episode during pregnancy or after delivery. These episodes were more severe than episodes occurring outside the perinatal or postpartum period. ${ }^{12}$ Furthermore, non-Dutch ethnicity is found to be associated with elevated pregnancy-specific anxiety. ${ }^{13}$ An explanation might be that women of non-Dutch ethnicity in general experience higher depression and anxiety levels owing to cultural differences, negative life experiences and lower socioeconomic status. $^{14}$

\section{Postpartum}

'Generation-R (and -Next)' is a Dutch populationbased prospective cohort study of mother-child dyads, running from pre-conception through adulthood. Of the 4941 women in this cohort, $8 \%$ developed postpartum depression. These women were statistically younger, less educated and more likely to be of non-Western origin than those without postpartum depression. Various pregnancy and delivery complications were predictive of postpartum depression, including pre-eclampsia, hospital admission during pregnancy and emergency Caesarean section. ${ }^{15}$ The prevalence of postpartum depression in The Netherlands is comparable to that in the USA and Europe. ${ }^{16,17}$ The global incidence of postpartum psychosis (not specific to The Netherlands) ranges from 0.89 to 2.6 in 1000 births. ${ }^{18}$ One Dutch study reports a postpartum relapse rate of $44.4 \%$ in patients with a history of psychoses limited to the postpartum period. This rate decreases to 0 for patients receiving lithium prophylaxis immediately postpartum. Women diagnosed with bipolar disorder are required to continue lithium prophylaxis during pregnancy and postpartum, thereby minimising the risk of relapse in those periods. ${ }^{19}$

\section{Perinatal and postpartum combined}

A Dutch population-based study reports a general increase of selective serotonin reuptake inhibitor (SSRI) usage throughout pregnancy and postpartum for the past 16 years. $^{20}$ In 2013-2014, $3.9 \%$ of women in The Netherlands used SSRIs before pregnancy, decreasing to $2.1 \%$ during pregnancy and increasing to $3.1 \%$ postpartum. These rates are comparable to those in Denmark and Italy. In the UK, rates of SSRI use among women during the year before pregnancy were noticeably higher $(8.8-9.6 \%) .{ }^{21}$ In the USA, prescription rates increased from $2.9 \%$ in 1999 to $10.2 \%$ in 2003 during pregnancy. ${ }^{22}$ The relatively lower prescription rates in The Netherlands imply a prescription threshold for SRRIs reflecting treatment only for more severe psychiatric disorders. One could suggest that low prescription rates imply limited ability of GPs to recognise perinatal mental illness. However, the presumption is that they refer pregnant women with mental health problems to 'POP care' for specialised treatment, including medication.

Mental healthcare during and after pregnancy has mainly focused on mothers. However, there is increasing awareness of the perinatal mental health of fathers in research worldwide. A meta-analysis estimated the prevalence of postpartum depression in fathers at $10.4 \%$, with maternal postpartum depression being the strongest predictor.

Nowadays, household structures are changing and this can contribute to psychopathology 

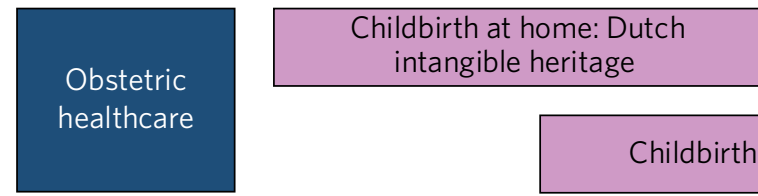

\section{Primary-care midwives supervised} $28 \%$ of all labours

\section{Childbirth at home $13 \%$}

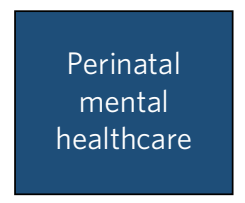

$8 \%$ postpartum depression

In-patient mother-baby units

POP-teams

Fig. 1

Key facts about The Netherlands and its perinatal mental healthcare system.

during the perinatal period. For example, being a single mother or bisexual/lesbian can increase the risk of depression and anxiety in pregnancy. ${ }^{23,24}$

\section{Service development and priorities}

Perinatal mental and infant health are topics of increasing government interest. The efficacy and cost-effectiveness of the national preventive programmes and biopsychosocial interventions that have been implemented to date are in need of evaluation.

\section{Current programmes and interventions}

Promising Start (Kansrijke Start: www.kansrijkestartnl.nl) is aimed at improving the mental and physical health of infants, focusing on the first 1000 days. The programme includes prenatal, perinatal and postpartum care and support for vulnerable parents (both current and prospective).

Not Pregnant Now $(\mathrm{Nu}$ Niet Zwanger: www.nunietzwanger.nl) provides financial (and other) support to vulnerable men and women helping them in their decision-making about sexuality, contraception and the desire to have children.

A programme from the Trimbos Institute is aimed at preventing perinatal and postpartum depression, including the early recognition of problems and the provision of psychoeducation to parents, in addition to counteracting stigmatisation by normalising problems (www.trimbos. nl/kennis/zwangerschap-en-depressie).

The campaign ' $\mathrm{Hi}$, it is okay' (Hey, het is oké: www.heyhetisoke.nl) also focuses on decreasing stigmatisation of mental health problems, including postpartum depression. Results show that people with psychological problems (anxiety and/or depression) felt less stigmatised after this national campaign.

In addition, midwives can use the standardised online Mind2Care questionnaire - provided and validated by LPKZ (the National Knowledge Centre for Psychiatry and Pregnancy) and containing questions about mental health, psychosocial problems and substance use - as a screening instrument to provide personalised recommendations for the provision/improvement of medical and psychosocial care during pregnancy and the postpartum period. $^{25}$ This instrument also includes questions from the Edinburgh Postnatal Depression Scale, which is validated in pregnant women in The Netherlands and also used as a separate screening instrument for depressive symptoms. ${ }^{26}$ The preventive implementation of this questionnaire for all pregnant women, including the measurement of response rates and identification of those not responding, could further optimise the accessibility of mental healthcare.

We think it is of great importance to translate mentioned initiatives into different languages and make those also available for low-literacy women.

Accessibility to help could be enhanced by e-health programmes. For example, the MamaKits Online intervention is aimed at reducing anxiety and depressive symptoms in pregnant women. ${ }^{27}$ The app WellMom is developed to improve welfare and prevent depressive feelings during and after pregnancy, and the app Loss is designed to amplify mental resilience of mothers and fathers who lost their child during 
or after pregnancy. Both apps are based on evidence-based effective interventions such as cognitive-behavioural therapy. For Turkish and Moroccan pregnant women with depressive symptoms, specifically, an online pilot course was developed and implemented called Positively Pregnant.

Worldwide, maternal and infant health mobile apps are increasingly being developed and used for health education. As mentioned, in The Netherlands, parents can download an app, produced by the Municipal Health Service (GGD), to make a lasting record of their child's development. The Centres for Disease Control and Prevention (USA) designed and launched a comparable app.

\section{Research and development}

As described above, both research on perinatal mental health and implemented interventions to improve mental health have mainly focused on mothers. Further research into support systems (especially co-parents) is of great importance. Psychosocial problems in families need a dyadic approach (mother-partner) rather than individual approach (mothers).

Fig. 1 summarises key facts about The Netherlands and its perinatal mental healthcare.

\section{COVID-19 pandemic and the impact on perinatal mental health services}

In December 2019, severe acute respiratory syndrome coronavirus 2 (SARS-CoV-2), which causes COVID-19, manifested in China and the spread of this virus was excessively rapid, resulting in a global pandemic. To prevent further dissemination in The Netherlands, strict measures were implemented in the daily lives of citizens: these included social distancing, working from home (if possible) and restricted travel. For pregnancy-related healthcare, restrictions were applied that included a limited amount of supportive accompanying persons for the pregnant women during consultations, labour and the postpartum period. During the pandemic mental healthcare is mainly provided by video- or phone consultation. However, it is know that the COVID-19 outbreak can trigger mental health problems such as anxiety, stress, anger, depressive symptoms and insomnia. ${ }^{28}$ The restrictive measures during pregnancy might cause additional perinatal stress, although we do not know the actual impact. In some countries, a mother with COVID-19 will be separated from her newborn to reduce the chance of transmission. This intervention may delay mother-infant attachment and can increase maternal anxiety. The separation of mother and child because of COVID-19 is not a standard of care in The Netherlands.

Knowledge about the COVID-19 virus and its effects on pregnancy is still growing. In general, pregnant women and the fetus are a vulnerable group concerning infectious diseases, owing to physiological changes in pregnancy. ${ }^{29}$ Therefore, pregnant women may have increased concerns about vertical transmission to their fetus, in addition to concerns about the health of their loved ones and their own health.

Research indeed shows an increase in symptoms of anxiety, depression and post-traumatic stress during the COVID-19 pandemic in both pregnant women and in new mothers during the postpartum period. ${ }^{30-32}$ Screening for perinatal mental health problems is therefore especially important at the moment, as this pandemic can be an additional stress factor. ${ }^{33}$

Owing to the COVID-19 pandemic, The Netherlands has been in national lockdown twice. Parents had to work at home if possible, while schools and day care were closed for children whose parents did not have a crucial job. COVID-19 has also caused economic devastation and created distance from community resources and support systems. These circumstances may stimulate domestic violence where it did not exist before, and may have worsened existing abuse in some families. In The Netherlands, no increase in official reports of domestic violence and abuse has been registered by Safe At Home. However, signs detected by childcare professionals indicate that emotional neglect of children increased during the first lockdown. ${ }^{34}$

The Dutch government provides reliable online information on COVID-19 and pregnancy. For instance, the website of the Covid Healthcare Support Centre (www.steunpuntcoronazorgen.nl/) focuses on the impact of COVID-19 pandemic (and the restrictions) on psychosocial factors: it provides information about mental stress specific to this pandemic and recommendations for support. Pharos (www.pharos.nl) provides information about COVID-19, related national measures and the impact on pregnancy and the postpartum period specifically for low-literacy citizens and for those whose native language is not Dutch.

\section{Conclusions}

Perinatal and postpartum mental health is a high priority for the Dutch government and healthcare professionals. The increasing interest in psychopathology during and after pregnancy has resulted in a well-developed biopsychosocial healthcare system in The Netherlands. One unique feature is the comprehensive collaboration between primary care (GPs and midwives) and hospital care (including POP care). Furthermore, the government organises and funds various healthcare programmes aimed at improving perinatal healthcare. The COVID-19 pandemic increases anxiety and depression during the perinatal period and further highlights the importance of a well-developed perinatal mental healthcare system. Research is needed to evaluate the programmes and forms of care, as well as to identify women who are not receiving appropriate healthcare. In addition, research should focus more on the psychopathology of the family as a whole rather than individuals only. 


\section{Data availability}

Data availability is not applicable to this article as no new data were created of analysed in this study.

\section{Author contributions}

I.L. contributed to the conception of the work, drafted the manuscript and approved the final version to be published, C.P.M.V., D.A.W., H.M. and M.J.T. contributed to the conception of the work, critically revised the manuscript for important intellectual content and approved the final version to be published. All authors agree to be accountable for all aspects of the work in ensuring that questions related to the accuracy or integrity of any part of the work are appropriately investigated and resolved.

\section{Funding}

This research received no specific grant from any funding agency, commercial or not-for-profit sectors.

\section{Declaration of interest}

None.

ICMJE forms are in the supplementary material, available online at https://doi.org/10.1192/bji.2021.47.

\section{References}

1 Watson R. Netherlands tops European Healthcare League, with UK Coming in at 12th. BMJ 2012; 344: e3430

2 Stronks K, Ravelli AC, Reijneveld SA. Immigrants in the Netherlands: equal access for equal needs? J Epidemiol Community Health 2001; 55: 701-7.

3 van der Gaag M, van der Heide I, Spreeuwenberg PM, Brabers AE, Rademakers J]. Health literacy and primary health care use of ethnic minorities in the Netherlands. BMC Health Serv Res 2017; 17(1): 350.

4 de Jonge A, Geerts C, Van Der Goes B, Mol B, Buitendijk S, Nijhuis J. Perinatal mortality and morbidity up to 28 days after birth among 743070 low-risk planned home and hospital births: a cohort study based on three merged national perinatal databases. BJOG 2015; 122: 720-8.

5 De Jonge A, Baron R, Westerneng M, Twisk J, Hutton EK. Perinatal mortality rate in the Netherlands compared to other European countries: a secondary analysis of Euro-PERISTAT data Midwifery 2013; 29: 1011-8.

6 Brownridge DA, Taillieu TL, Tyler KA, Tiwari A, Chan KL, Santos SC. Pregnancy and intimate partner violence: risk factors, severity, and health effects. Violence Against Women 2011; 17: 858-81.

7 Koirala P, Chuemchit M. Depression and domestic violence experiences among Asian women: a systematic review. Int Women Health 2020; 12: 21-33.

8 Landelijk Kenniscentrum Psychiatrie en Zwangerschap. "LKPZ, Van kinderwens tot ouderschap" ["LKPZ, From wish for a child to parenthood"]. LKPZ [National Knowledge Centre for Psychiatry and Pregnancy], 2021 (www.lkpz.nl).

9 de Graaf R, Ten Have M, van Gool C, van Dorsselaer S. Prevalence of mental disorders and trends from 1996 to 2009. results from the Netherlands mental health survey and incidence study-2. Soc Psychiatry Psychiatr Epidemiol 2012; 47: 203-13.

10 Pop V, Van Son M, Wijnen H, Spek V, Denollet J, Bergink V. Increase of depressive symptomatology during pregnancy over 25 years' time in four population based cohorts. J Affect Disord 2019; 259: 175-9.

11 Bennett HA, Einarson A, Taddio A, Koren G, Einarson TR. Prevalence of depression during pregnancy: systematic review. Obstet Gynecol 2004; 103: 698-709.

12 Meltzer-Brody S, Boschloo L, Jones I, Sullivan PF, Penninx BW The EPDS-Lifetime: assessment of lifetime prevalence and risk factors for perinatal depression in a large cohort of depressed women. Arch Women Ment Health 2013; 16: 465-73.

13 Westerneng M, Witteveen AB, Warmelink JC, Spelten E, Honig A, de Cock P. Pregnancy-specific anxiety and its association with background characteristics and health-related behaviors in a low-risk population. Compr Psychiatry 2017; 75: 6-13.

14 de Wit MA, Tuinebreijer WC, Dekker J, Beekman A-JT, Gorissen WH, Schrier AC, et al Depressive and anxiety disorders in different ethnic groups. Soc Psychiatry Psychiatr Epidemiol 2008; 43: 905-12.

15 Blom E, Jansen P, Verhulst F, Hofman A, Raat H, Jaddoe V, et a Perinatal complications increase the risk of postpartum depression: the Generation R study. BJOG 2010; 117: 1390-8.

16 O'Hara MW, Wisner KL. Perinatal mental illness: definition, description and aetiology. Best Pract Res Clin Obstet Gynaecol 2014: 28: 3-12.

17 Vesga-Lopez O, Blanco C, Keyes K, Olfson M, Grant BF, Hasin DS Psychiatric disorders in pregnant and postpartum women in the United States. Arch Gen Psychiatry 2008; 65: 805-15.

18 VanderKruik R, Barreix M, Chou D, Allen T, Say L, Cohen LS. The global prevalence of postpartum psychosis: a systematic review. BMC Psychiatry 2017; 17(1): 272.

19 Bergink V, Bouvy PF, Vervoort JS, Koorengevel KM, Steegers EA Kushner SA. Prevention of postpartum psychosis and mania in women at high risk. Am J Psychiatry 2012; 169: 609-15.

20 Molenaar NM, Lambregtse-van den Berg MP, Bonsel G]. Dispensing patterns of selective serotonin reuptake inhibitors before, during and after pregnancy: a 16-year population-based cohort study from the Netherlands. Arch Women Ment Health 2020; 23: 71-9.

21 Charlton R, Jordan S, Pierini A, Garne E, Neville A, Hansen A, et al Selective serotonin reuptake inhibitor prescribing before, during and after pregnancy: a population-based study in six European regions. BJOG 2015; 122: 1010-20.

22 Cooper WO, Willy ME, Pont SJ, Ray WA. Increasing use of antidepressants in pregnancy. Am J Obstet Gynecol 2007; 196: 544.e1-5.

23 Ross LE, Steele L, Goldfinger C, Strike C. Perinatal depressive symptomatology among lesbian and bisexual women. Arch Women Ment Health 2007; 10: 53-9.

24 Bogaerts AF, Devlieger R, Nuyts E, Witters I, Gyselaers W Guelinckx I, et al Anxiety and depressed mood in obese pregnant women: a prospective controlled cohort study. Obes Facts 2013; 6: 152-64

25 Quispel C. Psychopathology, psychosocial problems and substance use during pregnancy: screening and referral towards care (PhD thesis, Erasmus University Rotterdam, The Netherlands). Semantic Scholar, 2007: corpus ID 74688942.

26 Bergink V, Kooistra L, Lambregtse-van den Berg MP, Wijnen $\mathrm{H}$ Bunevicius R, Van Baar A, et al Validation of the Edinburgh Depression Scale during pregnancy. J Psychosom Res 2011; 70 385-9.

27 Heller HM, Hoogendoorn AW, Honig A, Broekman BF, van Straten A. The effectiveness of a guided internet-based tool for the treatment of depression and anxiety in pregnancy (MamaKits online): randomized controlled trial. J Med Int Res 2020: 22(3): e15172.

28 Torales J, O'Higgins M, Castaldelli-Maia JM, Ventriglio A. The outbreak of COVID-19 coronavirus and its impact on global mental health. Int J Soc Psychiatry 2020; 66: 317-20.

29 Dashraath P, Wong JLJ, Lim MXK, Lim LM, Li S, Biswas A, et a Coronavirus disease 2019 (COVID-19) pandemic and pregnancy. Am J Obstet Gynecol 2020; 222: 521-31.

30 Zanardo V, Manghina V, Giliberti L, Vettore M, Severino L, Straface G. Psychological impact of COVID-19 quarantine 
measures in northeastern Italy on mothers in the immediate postpartum period. Int J Gynecol Obstet 2020; 150: 184-8.

31 Wu Y, Zhang C, Liu H, Duan C, Li C, Fan J, et al Perinatal depressive and anxiety symptoms of pregnant women during the coronavirus disease 2019 outbreak in China. Am J Obstet Gynecol 2020; 223: 240.e1-9.

32 Basu A, Kim HH, Basaldua R, Choi KW, Charron L, Kelsall N, et al A cross-national study of factors associated with women's perinatal mental health and wellbeing during the COVID-19 pandemic. PloS One 2021; 16(4): e0249780.

33 Chen H, Selix N, Nosek M. Perinatal anxiety and depression during COVID-19. J Nurse Pract 2021; 17: 26-31.

34 Vermeulen S, van Berkel S, Alink L. Kindermishandeling Tijdens de eerste Lockdown [Child Abuse during the First Lockdown]. University of Leiden, 2020 